\title{
Nanofibrous Composite Materials Integrating Nano/Micro Particles between the Fibres
}

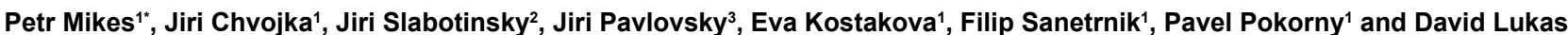

${ }^{1}$ Technical University of Liberec, Czech Republic

${ }^{2}$ National Institute for NBC Protection, Kamenna, Czech Republic

${ }^{3} \mathrm{VŠB}-T e c h n i c a l$ University of Ostrava, Czech Republic

\begin{abstract}
This article deals with the continual incorporation of particles by the ultrasonic dispersion in situ into a nanofibrous matrix produced by the electrospinning process. The new technology is based on the use of the needleless electrospinning method in combination with the ultrasound-enhanced dispersion of sub-micro or micro particles, which are deposited between nanofibres onto the support material. The main advantage of this technology is the independence of particle-incorporation of the electrospinning process. The particles are trapped between the fibres and they remain uncovered by polymer, thus maintaining all their active properties. Such materials can be cut with scissors without the particles being released. In this paper the authors present figures from scans of the electron microscopy of the newly-designed nanocomposite material and its morphological analysis, such as the particle distribution. The material was used as a sorbent of bis(2-chlorethyl) sulfide (mustard gas) with a sorption time greater than 240 minutes. Such material has been developed to be used for protection against chemical warfare agents; yet, it can be employed for several other applications depending on the powder material dispersed onto the nanofibrous layer.
\end{abstract}

Keywords: Needleless electrospinning; Particles; Ultrasound; Nanocomposites; Mustard gas

\section{Introduction}

Nanofibrous materials can be produced by the well-known needle [1] or needleless [2,3] electrospinning processes. Composite materials based on electrospun nanofibres are at the forefront of the modern material science. There are several methods for incorporating particles [4-10] into the nanofibrous materials, e.g. electrospinning of dispersed particles, such as fullerenes [6], nanotubes [8,9] or hydroxyapatite [7] from polymeric solutions, core-shell electrospinning [4,5] or a combination of the electrospinning and electrospraying processes [10]. But all of these methods are somehow limited, mainly by the amount of the powder material that can be incorporated into the nanofibres. The viscosity of the polymeric solution is increased by addition of any powder material, which affects the electrospinning process [6-9].

The task for the presented investigation was to develop a new type of material for sorption of mustard gas and for other applications, depending on the powder used for the process. The achieved outcome was the development of a new method for integration of particles inbetween the nanofibres instead of into the fibres themselves.

In contrast to the other methods mentioned above, the particles in this experiment were dispersed by ultrasonic vibrations and then covered with the deposited nanofibres directly after they fell on the collector. This means that the particles are trapped in-between the fibres, which enables a unique incorporation of a higher density of particles. The size of the particles plays an important role in the selection of the process of their incorporation. Furthermore, the applications of particles depend on the material from which they are made. Taking all of that into consideration, the authors prepared membranes containing active carbon particles trapped by nanofibres and tested them for the sorption of the mustard gas.

In the second chapter the methods and materials used in this experiment are described together with the newly-produced materials, their analysis and their usage potential, as revealed by their tests for mustard gas penetration.

\section{Materials and Methods}

For the electrospinning process, a 10\% aqueous polyvinyl alcohol (PVA) solution was prepared by dissolving the polymer in distilled water. The company Novácké chemické závody, Nováky in Slovakia provided the PVA with a predominant molecular weight of $60.000 \mathrm{amu}$. Crosslinking of the PVA nanofibres was achieved by adding $3 \mathrm{wt} . \%$ of 99\% phosphoric acid and $4 \mathrm{wt} . \%$ of Glyoxal, both of which are produced by Sigma Aldrich. The nanofibrous layer was then heated to $135^{\circ} \mathrm{C}$ for 5 minutes to activate the crosslinking process. Next, a polymeric solution for the electrospinning process was prepared from two components. Kuraray America, Inc. produced polyvinyl butyral (PVB), Mowital ${ }^{\circ} \mathrm{B}$ $60 \mathrm{H}$, with an average molecular weight of $60.000 \mathrm{amu}$; it was dissolved in a $10 \mathrm{wt} . \%$ solution in ethanol-water $(9: 1 \mathrm{v} / \mathrm{v})$.

The nanofibres were collected on the spunbond polypropylene produced by Pegas Nonwovens, Czech Republic. The distribution of the fibre diameter lengths was in the range from 120 to $380 \mathrm{~nm}$ with an average of $200 \mathrm{~nm}$. A histogram can be seen in Figure 1A. The NIS Elements software produced by Nikon was used for calculations of the average inter-fibrous distance, which was identified as being $(1.67 \pm$ 0.69) $\mu \mathrm{m}$.

The particles of active carbon Norit GL Extra were milled by RETCH CryoMill with an average diameter at $2.8 \mu \mathrm{m}$ and deviation of $\pm 1.5 \mu \mathrm{m}$. The particle size distribution depended on the duration of

*Corresponding author: Petr Mikes, Technical University of Liberec, Czech Republic, Tel: 00420485353230; E-mail: petr.mikes@tul.cz

Received February 01 2016; Accepted March 01, 2016; Published March 06 , 2016

Citation: Mikes P, Chvojka J, Slabotinsky J, Pavlovsky J, Kostakova E, et al. (2016) Nanofibrous Composite Materials Integrating Nano/Micro Particles between the Fibres. J Membra Sci Technol 6: 146. doi:10.4172/2155-9589.1000146

Copyright: ( 2016 Mikes $\mathrm{P}$, et al. This is an open-access article distributed under the terms of the Creative Commons Attribution License, which permits unrestricted use, distribution, and reproduction in any medium, provided the original author and source are credited. 
milling. The average diameter distribution was measured by Zetasizer Nano ZS Malvern UK - model ZEN3601 and can be seen in Figure 1B.

A schematic diagram and photography of the setup used are shown in Figures $2 \mathrm{~A}$ and $2 \mathrm{~B}$, respectively. There are several technological parameters that always have to be controlled for different materials, such as air humidity, concentration of the polymer in the solution for the electrospinning process and the amplitude and frequency for the particle dispersion. The details of this setup are described below.

For the needleless electrospinning process the investigators used positive and negative high voltage sources, a $300 \mathrm{~W}$, high voltage DC power supply with regulators, model number SL 150, manufactured by Spellman High Voltage, Inc. with output parameters of $0-50 \mathrm{kV}, 6$ $\mathrm{mA}$. The positive voltage of $35 \mathrm{kV}$ was applied to the roller (spinning electrode) and the negative voltage of $5 \mathrm{kV}$ to the belt collector. The positive voltage was set to reach the critical voltage [2] and collector was negatively charged to increase the potential difference and to support the collection of fibers preferentially to the belt collector. The distance between the spinner and the collector was kept at $15 \mathrm{~cm}$ which is the optimal distance for fibrous elongation and solvent evaporation.

Nanofibres were collected on the belt collector, which consists of a motor powering two cylinders positioned $40 \mathrm{~cm}$ apart and holding the supported non-woven textile. The rotational speed of the gearbox powering the belt collector was $10 \mathrm{rpm}$ and the belt was $80 \mathrm{~cm}$ long and $20 \mathrm{~cm}$ wide. First of all simple nanofibrous layer without any particles was collected and then the belt collector was twisted three times while both electrospinning and ultrasonic dispersion processes were in progress. Finally the composite was covered by another additional nanofibrous layer. Both inner and outer nanofibrous layer was prepared for better manipulation with the material.

This technology is relatively robust because the processes are independent of each other. The electrospinning parameters, such as voltage, humidity, the distance between the collector and the electrode, the concentration, the molecular weight of the polymer used, etc.,

A

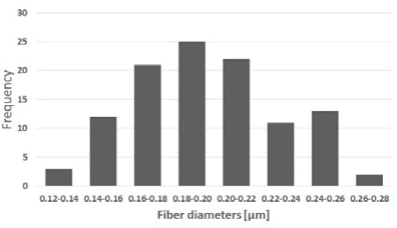

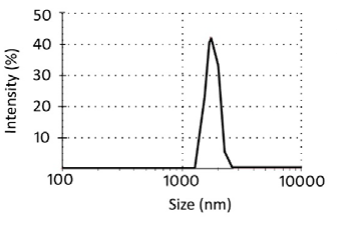

Figure 1: A) The distribution of nanofibrous diameters measured by NIS element software and B) size distribution of the milled particles used for the ultrasonic spraying measured by the dynamic light scattering Zetasizer ZEN3601.

$\$$

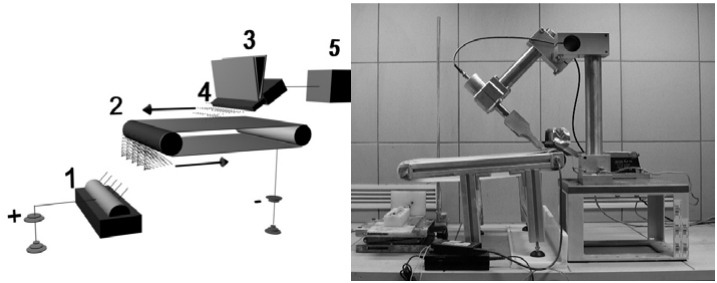

Figure 2: Schematic diagram (A) and photograph (B) of experimental setup for the creation of nanocomposite materials, which consists of (1) needleless spinning electrode, (2) belt collector, (3) particle conveyor, (4) ultrasound sonotrode, and (5) feeder. have already been well described [1,2]. The ultrasonic dispersion is completely independent of the electrospinning process.

The most difficult problem to solve was the formation of a homogeneous distribution of the dispersed particles. Van der Waals forces between particles increase, but the particle diameter decreases and the particles tend to agglomerate [11]. The use of ultrasonic vibrations is necessary for the dispersion of these clusters [12] and for the creation of a more homogeneous type of material. Particles were de-agglomerated by the $18-\mathrm{cm}$-wide and $1-\mathrm{cm}$-thick dural sonotrode produced by Ultratech Company. A Sonic Digital ULC generator produced by Weber Ultrasonics with the power of $400 \mathrm{~W}$ and frequency of $20 \mathrm{kHz}$ was used to power the sonotrode.

The particles were stored in a reservoir and continually conveyed by a series of vibrations to the edge of the sonotrode. The conveying device was specially designed for this technology. The device is powered by the Afag HLF 12 feeder controlled by the PSG1 unit with the vibrations amplitude with the constant frequency of $220 \mathrm{~Hz}$. The relationship between the amplitude and the amount of the conveyed particles can be seen in Figure 3. The particles were subsequently covered by nanofibres. The temperature during the experiments was $21 \pm 5^{\circ} \mathrm{C}$ and the relative humidity $65 \pm 5 \%$. A sieve shaker (Retsch AS 200) was used for testing the particles bonding to the fibres (Figure 3).

The thickness of the produced layer was controlled by the running time of the process and by the size of the particle dosage into the nanofibrous layer.

\section{Adsorption ability of the nanocomposite membrane}

A nanofibrous material with incorporated particles of active carbon was tested for penetration of CWA. Ninety-six-per-cent-pure bis(2chlorethyl) sulfide, or mustard gas was applied in the liquid phase on a membrane made of High Density Polyethylene (HDPE) with the thickness of $30 \mu \mathrm{m}$ [13]. The mustard gas vapour penetrating through the HDPE was adsorbed by the particles of active carbon. The sorption efficiency on the reverse side of the sample was evaluated by chemical indication factors caused by the degree of penetration and by gas chromatography. The chemical indication was based on the reaction of the penetrating mustard gas with a paper indicator saturated with chloramine (N-chlorobenzoic-o-toluidine) (Figure 4). The change of the $\mathrm{pH}$ value was indicated by Congo red [13]. The gas chromatography used the flame-ionizing indication on a Carousel 2000 device to measure the mustard gas permeation [14]. The chemical sensitivity



Figure 3: The relationship between the amplitude and the amount of the conveyed particles. 
indication was $0.1 \mu \mathrm{g}$ of mustard gas. The results were measured in terms of the time taken for the vapours to penetrate to the reverse side, which is the breakthrough time (BT) (Table 1). The progress of permeation was characterized by the speed of penetration all the way through to the back side, F, and the total penetrated amount of mustard gas, labelled as Q.

\section{Results and Discussion}

The produced nanofibrous composite material has unique properties due to its original and innovative structure. The particles are trapped in-between fibres and do not get covered by any polymer. The material can therefore keep its characteristics, such as, for example, high porosity in the case of active carbon particles [15-18]. The interfibrous distances within the nanofibrous matrix enable even submicron particles to be incorporated [19].

The average areal density of the nanofibres was $5 \mathrm{~g} \cdot \mathrm{m}^{-2}$ and the areal density of particles reached up to $20 \mathrm{~g} . \mathrm{m}^{-2}$ within 10 minutes. These values were measured independently for both processes by weighing the electrospun polymer and the dispersed particles. The amount of particles was controlled by the amplitude of the generator (Figure 3). The scanning electron microscope (SEM) images of the active carbon incorporated in-between the electrospun nanofibres are shown in Figures 5A-5D.

The natural phenomenon of activated carbon particles is their random agglomeration [18-26]. The use of ultrasound proved to be a good method for the dispersion of the dry particles into the nanofibrous matrix [12]; however, some particles remained agglomerated. It was mainly due to the imperfect positioning of the sonotrode and

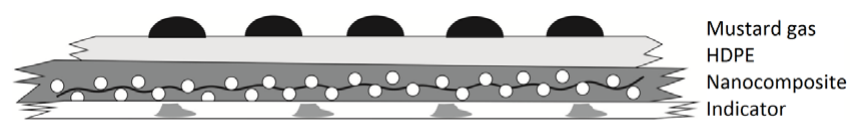

Figure 4: The setup for measuring the chemical indication of mustard gas (HD) where HDPE is the High Density Polyethylene membrane which covers the nanocomposite membrane with the indicator on its back.
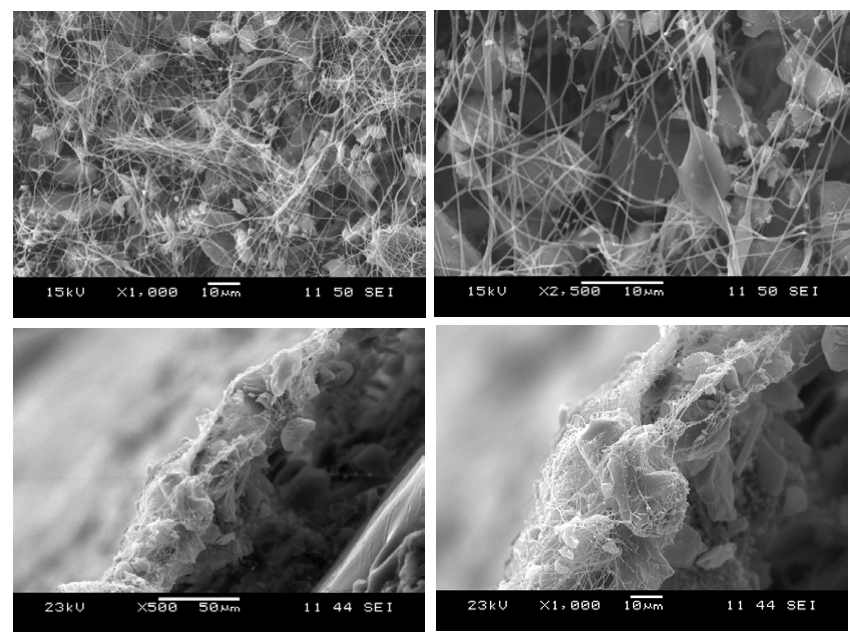

Figure 5: A-D) Scanning electron micrographs of electrospun nanofibrous material with particles of active carbon dispersed into the PVA nanofibrous layer. Figures $5 \mathrm{~A}$ and $5 \mathrm{~B}$ are standard SEM pictures of the face of the layer; Figures $5 \mathrm{C}$ and $5 \mathrm{D}$ are the edges of the layer. conveyor, which is why some particles fell onto the web undispersed. The homogeneity of particles distribution is important for sorption of mustard gas. The agglomeration creates places with lower amount of active carbon particles where gas can penetrate through the membrane easily.

This technology can be used for many different combinations of polymeric electrospun nanofibres and submicron or micro particles.

Using a bi-component spunbond material made from polypropylene and polyethylene fibres could thermally laminate the produced layer. Polyethylene has a lower melting temperature than polypropylene.

The produced materials were tested for particles released from the nanofibrous matrix by using a Retsch AS 200 sieve shaker. The materials were placed on the sieve with a mesh size of $1 \mathrm{~mm}$. The sample was weighed both before and after shaking with the amplitude of $80 \%(2.4$ $\mathrm{mm}$ ) for ten minutes. The average amount of loosened particles was 3\% from the total weight of all particles.

From the SEM pictures in Figures 5A-5D it is clearly seen that particles of active carbon have been trapped by the fibres. The nanofibrous web prevents their releasing even after it is cut, thus particles stay bonded to the layer. Some particles remained agglomerated due to the above mentioned reason. Samples used for SEM didn't have any additional covering nanofibrous layer for better observation of bonded particles.

\section{Measurement of sorption ability of the resultant material}

Table 1 shows the positive effect of active carbon particles compared to the pure HDPE and also to a composite consisting of HDPE, nanofibres and the spunbond material. Not only does the amount of the activated carbon, which is responsible for the efficiency of the protective system, play a role, but also its areal distribution and homogeneity have their significant influence. It can be seen that the maximum breakthrough time (BT) was identified for the HDPE+PVB+AC 16 material. The prolonged time of mustard gas penetration in the fabric will increase its safety.

Even more conclusive results can be seen in Figure 6, where there are almost no differences in the speed of mustard gas penetration. There is a significant decrease in penetration for all composites in comparison to the HDPE membrane. However, the penetration continues even after the gas flow does not influence it any further directly. This is probably caused by the uneven distribution of the sorbent particles in the composite material. Determination of specific surface area at the prepared materials will influence the penetration of mustard gas, as can be seen in Table 2 .

It is evident that there is a continuous increase of adsorption of the mustard gas due to the use of the given composite, as shown by the values of $\mathrm{Q}$ for the HDPE and the composites in different time intervals. However there is almost no difference between all materials in terms of total penetration $\mathrm{Q}$ and penetration rate $\mathrm{F}$. This is probably due to the sorption ability of activated carbon particles trapped by nanofibers. It

\begin{tabular}{|c|c|c|}
\hline Composition & Active carbon content [wt. \%] & BT [min] \\
\hline HDPE & - & 4 \\
\hline HDPE+PVA & - & 4 \\
\hline HDPE+PVA+AC 8 & 8 & $285 \pm 45$ \\
\hline HDPE+PVA+AC 16 & 16 & $313 \pm 60$ \\
\hline HDPE+PVB+AC 16 & 16 & $360 \pm 60$ \\
\hline
\end{tabular}

Table 1: Breakthrough time (BT) of the composite membrane against mustard gas penetration at $25^{\circ} \mathrm{C}$. 


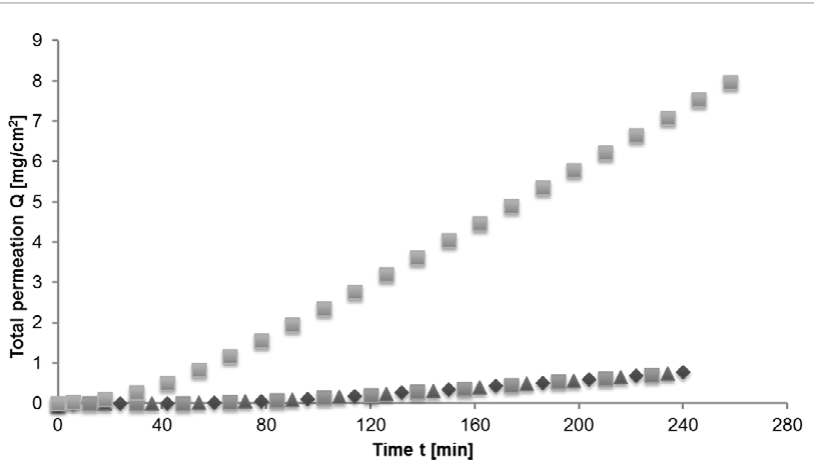

- $\mathrm{HDPE}+\mathrm{PVA}+\mathrm{AC} 8 \backsim \mathrm{HDPE}+\mathrm{PVA}+\mathrm{AC} 16 \triangle \mathrm{HDPE}+\mathrm{PCL}+\mathrm{AC} 16=\mathrm{HDPE} 0.030 \mathrm{~mm}$

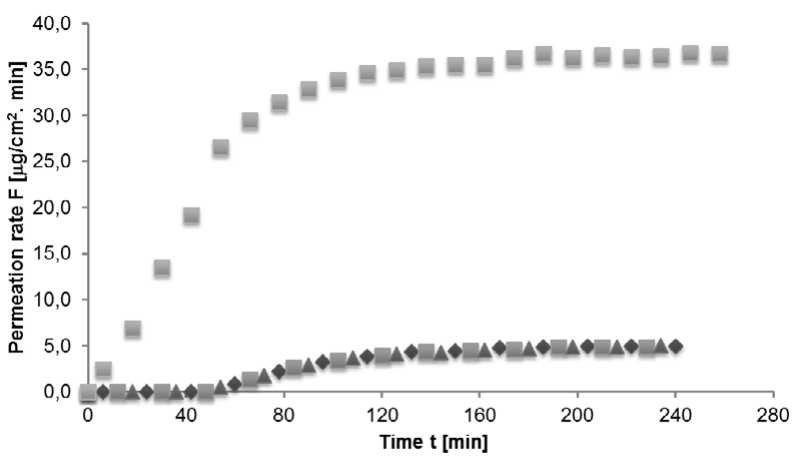

-HDPE+PVA + AC 8 «DPE+PVA + AC $16 \triangle \mathrm{HDPE}+\mathrm{PVB}+\mathrm{AC} 16 \approx \mathrm{HDPE} 0.030 \mathrm{~mm}$

Figure 6: The total amount of mustard gas penetrating to the back of the HDPE and composites at $30^{\circ} \mathrm{C}$ (above) and the rate of penetration of mustard gas through the HDPE and composites at $30^{\circ} \mathrm{C}$, where $\mathrm{AC} 8$ and $\mathrm{AC} 16$ indicate the amount of active carbon in the composite (Table 2).

is evident mainly for HDPE+PVA+AC8 and HDPE+PVA+AC16. Both materials showed the same time of sorption as can be seen on Figure 6. The question still remains how it will be for longer time of permeation. But the time of operation of apparatus was limited. Anyway, for its use as a protection membrane against chemical warfare, the time of sorption up to $240 \mathrm{~min}$ is sufficient. Therefore the lower amount of activated carbon particles with $8 \mathrm{wt} . \%$ is ample.

Prepared materials were also analysed using Sorptomatic series 1990 apparatus, Thermo Quest (CE Instruments), Rodano (Milano) with the method of low-temperature adsorption of nitrogen molecules (boiling point $77.7 \mathrm{~K}$ ), from a vacuum into atmospheric pressure for determination of specific surface area. It was revealed that the specific surface area was lower for the composite materials in comparison with the activated carbon particles (AC). Also no significant specific surface area differences were measured by the BET analysis for the material $\mathrm{HDPE}+\mathrm{PVA}+\mathrm{AC}$ irrespective to the amount of activated carbon (Table 2). The situation is different for the material HDPE+PVB+AC 16 (16 wt.\% of $\mathrm{AC}$ ), where the specific surface area is approximately two times higher than for HDPE+PVA+AC 16 (16 wt.\% of AC). The reason for the difference in higher specific surface of nanofibers with PVB against nanofibers with PVA should be its roughness. Higher content of activated carbon (AC) and its incorporation in-between the electrospun nanofibers thus increases the specific surface area and also more contact points between individual fibers, as shown in Figure 5. Larger specific surface areas can provide sufficient contact between HDPE+PVB+AC 16 and mustard gas (Table 1) and this leads

\begin{tabular}{|c|c|}
\hline Specimen & specific surface area ${ }^{\left[{ }^{2} \cdot \mathbf{g}^{-1}\right]}$ \\
\hline Active carbon (AC) & $1274.0^{+}$ \\
\hline HDPE+PVA+AC 8 & 164.2 \\
\hline HDPE+PVA+AC 16 & 148.7 \\
\hline HDPE+PVB+AC 16 & 291.3 \\
\hline
\end{tabular}

Table 2: Specific surface area of active carbon and composites. The specific surface area of microporous material (active carbon) evaluated by using the Langmuir method [16] is labelled by + .

to increase of the BT value [18].

Electrospinning technique has many remarkable characteristics, such as fine diameters ranging from submicron to several nanometers [19], large specific surface area, high porosity and high permeability [20] etc. Simultaneously, recent studies have shown that different properties such as surface area and porosity become more significant when the fibres diameter falls below $20 \mathrm{~nm}$. An ideal fiber diameter is below $20 \mathrm{~nm}$ for optimal performances, however electrospun fibers from the traditional process typically have a diameter in the range $100-500 \mathrm{~nm}$ [21]. Electrospun nanofibers are featured with very large surface area per unit mass, small pore size a more contacts between individual fibers. Electrospun nanofibers with active carbon have large surface area (Table 2) which is in agreement with Saeed [22].

The objective is a robust method for manufacturing extremely small nanofibers in large quantities and with a uniform size. Recently, the novel two-dimensional (2D) nanofibers were generated in 3D fibrous mats by optimizing processing parameters in electrospinning [23].

In Table 2 the specific surface area studied by the BET [15] theory and for active carbon (AC) by the Langmuir theory [16] is presented.

The final step of the investigation was the performance of the SEM observation of the nanofibrous composite layer after the sorption of mustard gas. Samples were decontaminated by washing in flowing air with the temperature $25-30^{\circ} \mathrm{C}$. As can be seen in Figures $7 \mathrm{~A}$ and $7 \mathrm{~B}$, nanofibres slightly swell their volume by sorption of mustard gas but without any visible damage to their structure. The material, which was used for sorption of mustard gas, was finally covered by a thin layer of nanofibres to prevent particles release while being manipulated. Therefore not many particles are visible in Figures 7A and 7B.

As the final result of this study, the use of PVB is a better choice compared to PVA. The amount of activated carbon particles is sufficient with $8 \mathrm{wt} . \%$ for time of sorption lower than $240 \mathrm{~min}$. Therefore, the optimal material should combine PVB nanofibers with 8 wt.\% of activated carbon.

\section{Conclusions}

The materials presented here can be used for a number of technical applications. In the investigation they were successfully tested for the sorption of chemical substances, such as mustard gas. The direct action of the liquid mustard gas through the High Density Polyethylene gave high values of breakthrough time for the composites, but not for the membrane without sorbents. It is already possible to achieve such results with a relatively small amount of active carbon.

The technology developed in the investigation proved to be applicable for different powder materials. Its main advantage is the fact that the particles stay uncovered by the polymeric matrix and they keep their unique properties. They are simply trapped by the adhesion forces of the nanofibres, which enables the integration of even submicron particles depending on the inter-fibrous porosity. It is as well possible to combine particle materials and electrospun polymers and to create 

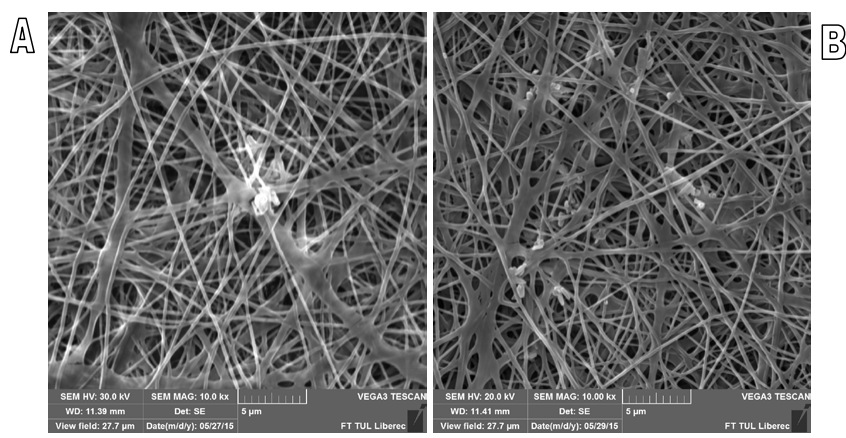

Figure 7: Scanning electron microscope pictures of tested samples of HDPE+PVA+AC16, A) before and B) after mustard gas sorption.

a tailor-made product. Another advantage of these materials is that they can be cut by standard methods (scissors, scalpel, razor blade, etc.), which makes them widely applicable depending on the powder material integrated into the composite.

The composite material with particles was successfully tested for sorption of the mustard gas with time of sorption more than 300 minutes. However, some aspects of the material have to be improved, mainly the homogeneity of the dispersed material. This was evident for the active carbon particles. Active carbon is a very hygroscopic material and therefore its agglomeration increases during the process. The homogeneity can be improved, e.g. by use of some more closed system with air conditioning, which prevents the agglomeration of particles. Another possibility to improve the homogeneity of the particles distribution is the presence of some suction system under the nanofibrous layer.

\section{Acknowledgment}

Support for this research was provided by the Ministry of the Interior of the Czech Republic, BV II/2-VS, Nanomaterials for personal protection against CBRN substances". This paper was supported by the project of. Regional Material Technological Research Centre - Sustainability programme“, registration number LO1203.

\section{References}

1. Reneker DH, Yarin AL (2008) Electrospinning jets and polymer nanofibers. Polymer 49: 2387-2425.

2. Lukas D, Sarkar A, Pokorny P (2008) Self-organization of jets in electrospinning from free liquid surface - a generalized approach. J Appl Phys 103: 1-7.

3. Jirsak O, Sanetrnik F, Lukas D, Kotek V, Martinova L, et al. (2005) A method of nanofibres production from a polymer solution using electrostatic spinning and a device for carrying out the method, US Patent WO2005024101.

4. Moghe AK, Gupta BS (2008) Co-axial electrospinning for nanofiber structures: Preparation and applications. Polym Rev 48: 353-377.

5. Mickova A, Buzgo M, Benada O, Rampichova M, Fisar Z, et al. (2012) Core/ Shell Nanofibers with Embedded Liposomes as a Drug Delivery System. Biomacromolecules 4: 952-962.

6. Yang P, Zhan S (2011) The fabrication of PPV/C60 composite nanofibers with highly optoelectric response by optimization solvents and electrospinning technology. Materials Letters 3: 537-539.

7. Kim GM, Asran ASh, Michler GH, Simon P, Kim JS (2005) Electrospun PVA HAp nanocomposite nanofibers: biomimetics of mineralized hard tissues at a lower level of complexity. Bioinspir Biomim 3: 046003.
8. Kannan P, Eichhorn SJ, Young RJ (2007) Deformation of isolated single-wall carbon nanotubes in electrospun polymer nanofibres. Nanotechnology 18: 235707.

9. Kostakova E, Meszaros L, Gregr J (2009) Composite nanofibers produced by modified needleless electrospinning. Materials Letters 28: 2419-2422.

10. Jaworek A, Krupa A, Lackowski M, Sobczyk AT, et al. (2008) Nanocomposite fabric formation by electrospinning and electrospraying technologies. J Electrostat 67: 435-438.

11. Hartley PA, Parfitt GD, Pollack LB (1985) The role of the van der Waals force in the agglomeration of powders containing submicron particles. Powder Technology 1: 35-46

12. Sauter C, Emin MA, Schuchmann HP, Tavman S (2008) Influence of hydrostatic pressure and sound amplitude on the ultrasound induced dispersion and deagglomeration of nanoparticles. Ultrasonics Sonochemistry 15: 517-523.

13. MAZL3/95. Estimation of breakthrough time of mustard drops through protective materials at static conditions. Accredited Method National Institute for NBC Protection of the Czech Republic.

14. MAZL 39-10/Permeatest 4. Determination of resistance membrane material to penetration of mustard by gas chromatography. Accredited Method National Institute for NBC Protection of the Czech Republic.

15. Brunauer S, Emmet PH, Teller EJ (1938) Adsorption of gases in multimolecular layers. J Am Chem Soc 60: 309.

16. Langmuir I (1918) The adsorption of gases on plane surfaces of glass, mica and platinum. J Am Chem Soc 40: 1361

17. Dror $Y$ (2003) Carbon nanotubes embedded in oriented polymer nanofibers by electrospinning. Langmuir 19: 7012.

18. March H, Rodriguez-Reinoso F (2006) Actived Carbon. Elsevier, Oxford, UK

19. Eichhorn SJ, Sampson WW (2005) Statistical geometry of pores and statistics of porous nanofibrous assemblies. J R Soc Interface 2: 309-318.

20. Tingting L, Zhiming Z, Wei L, Ce L, Jianfei W, et al. (2016) H4SiW12O40/ polymethylmethacrylate/polyvinyl alcohol sandwichnanofibrous membrane with enhanced photocatalytic activity. Colloids Surf A Physicochem Eng Asp 489: 289-296.

21. Buchko CJ, Chen LC, Shen Y, Martin DC (1999) Processing and microstructura characterization of porous biocompatible protein polymer thin films. Polymer 40: 7397-7407.

22. Saeed K, Haider S, Oh TJ, Park SY (2008) Preparation of amidoxime-modified polyacrylonitrile (PAN-oxime) nanofibers and their applications to metal ions adsorption. J Memb Sci 322: 400-405.

23. Huang CB, Chen SL, Lai CL, Reneker DH, Qiu H, et al. (2006) Electrospun polymer nanofibres with small diameters. Nanotechnology 17: 1558-1563.

24. Li D, McCann JT, Xia Y (2005) Use of electrospinning to directly fabricate hollow nanofibers with functionalized inner and outer surfaces. Small 1: 83-86

25. Ding B, Li C, Miyauchi Y, Kuwaki O, Shiratori S (2006) Formation of novel 2D polymer nanowebs via electrospinning. Nanotechnology 17: 3685-3691.

26. Anson M, Marchese J, Garis E, Ochoa N, Pagliero C (2004) ABS copolymeractivated carbon mixed matrix membranes for $\mathrm{CO} 2 / \mathrm{CH} 4$ separation. J Memb Sci 243: 19-28. 